INPLASY

PROTOCOL

To cite: Fan et al. Hyperbaric Oxygen Therapy for treating chronic diabetic foot ulcers : an Overview of Systematic Reviews. Inplasy protocol 202110008. doi:

10.37766/inplasy2021.1.0008

Received: 3 January 2021

Published: 3 January 2021

Corresponding author: Weijing Fan

18811023202@163.com

Author Affiliation:

Shuguang Hospital Affiliated to

Shanghai University of

Traditional Chinese Medicine

Support: No.81774310;

81804096.

Review Stage at time of this submission: The review has not yet started.

Conflicts of interest:

None.

\section{Hyperbaric Oxygen Therapy for treating chronic diabetic foot ulcers : an Overview of Systematic Reviews}

\author{
Fan, W1; Li, W2; Huang, R³; Liu, G4; Fu, C5; Yang, B6.
}

Review question / Objective: An Overview of Systematic Reviews was completed to evaluate the clinical evidence available on HBOT for DFUs.

Condition being studied: A number of systematic reviews related to hyperbaric oxygen therapy in the treatment of diabetic foot have been published, but the conclusions are not unified, and there are no studies to integrate the relevant clinical evidence and systematically evaluate the quality of evidence.

Information sources: PubMed, Cochrane Library, Web of knowledge, Embase, MEDLINE, Chinese National Knowledge Infrastructure Database; the Chongqing VIP Database; Chinese Biomedical Database; Wanfang Database.

INPLASY registration number: This protocol was registered with the International Platform of Registered Systematic Review and Meta-Analysis Protocols (INPLASY) on 3 January 2021 and was last updated on 3 January 2021 (registration number INPLASY202110008).

\section{INTRODUCTION}

Review question / Objective: An Overview of Systematic Reviews was completed to evaluate the clinical evidence available on HBOT for DFUs.
Condition being studied: A number of systematic reviews related to hyperbaric oxygen therapy in the treatment of diabetic foot have been published, but the conclusions are not unified, and there are no studies to integrate the relevant clinical 
evidence and systematically evaluate the quality of evidence.

\section{METHODS}

Search strategy: We will identify SRs through systematic searches of the following databases: PubMed, Cochrane Library, Web of knowledge, Embase, MEDLINE, Chinese National Knowledge Infrastructure Database; the Chongqing VIP Database; Chinese Biomedical Database; Wanfang Database. The searching time is limited from the establishment time of all databases to December 1, 2020. In addition, ongoing and unpublished systematic reviews will be searched in PROSPERO. In addition, we will manually search all references included in the literature.

Participant or population: Diagnosed with diabetic foot, both ischemic and nonischemic ulcers, regardless of sex, age, course of disease, source of case, country or race. They may have other chronic disease.

Intervention: Hyperbaric oxygen therapy alone or in combination with other conventional therapy.

Comparator: Conventional treatment (standard wound care) or sham intervention.

Study designs to be included: All systematic reviews (SRs) of randomizedcontrolled clinical trials and notrandomized clinical trials.

Eligibility criteria: Diagnosed with diabetic foot, both ischemic and non-ischemic ulcers, regardless of sex, age, course of disease, source of case, country or race. They may have other chronic disease.

Information sources: PubMed, Cochrane Library, Web of knowledge, Embase, MEDLINE, Chinese National Knowledge Infrastructure Database; the Chongqing VIP Database; Chinese Biomedical Database; Wanfang Database.
Main outcome(s): Primary outcomes: Ulcer healing rate, Incidence of major amputation, Incidence of minor amputation.

Additional outcome(s): Secondary outcomes: Ulcer healing time, Ulcer area reduction rate, Percutaneous oxygen partial pressure, Quality of life score, pain score.

Quality assessment / Risk of bias analysis: AMSTAR2 is used to evaluate the methodological quality of included systematic reviews. AMSTAR 2 checklist contains $\mathbf{1 6}$ items, of which 7 are critical (2, $4,7,9,11,13,15)$. The overall evidence quality of each systematic review is divided into four categories, rated "high" if only one or more non-critical items are not satisfied, "medium" if more than one noncritical item is not satisfied, "low" if only one key item is not satisfied, and "very low" if one or more key items are not satisfied.

Strategy of data synthesis: GRADE was used to evaluate the quality of evidence of hyperbaric oxygen therapy in the treatment of diabetic foot. Te following criteria were taken into account: risk of bias (that is study limitations), inconsistencies, indirectness, inaccuracy, and publication bias.

Subgroup analysis: No.

Sensibility analysis: No.

Language: No restriction.

Country(ies) involved: China.

Keywords: Hyperbaric Oxygen Therapy; chronic diabetic foot ulcers; Overview; Systematic Reviews.

Contributions of each author:

Author 1 - Weijing FAN.

Author 2 - Wenhui LI.

Author 3 - Renyan HUANG.

Author 4 - Guobin LIU.

Author 5 - Changgeng FU.

Author 6 - Baozhong YANG. 